\title{
A compact PET detector module using SiPMs and MVT digitizers
}

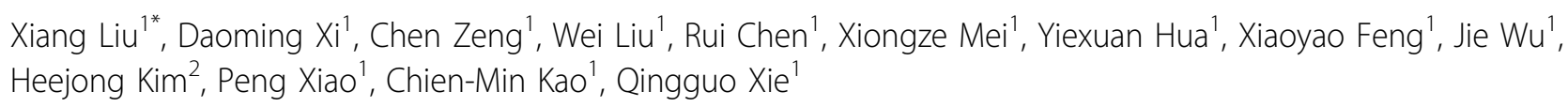

From PSMR 2015: 4th Conference on PET/MR and SPECT/MR

La Biodola, Isola d'Elba, Italy. 17-21 May 2015

${ }^{1}$ Huazhong University of Science \& Technology, Hubei, China
Nowadays the simultaneous PET/MRI is being paid more and more attentions. Since MRI provides a high resolution, unsurpassed soft tissue contrast imaging and PET reveal the metabolism progress in molecular level, it is reasonable to believe the PET/ MRI will be a powerful tool to improve the understanding of pathogenesis and mechanism of brain disease. Such a simultaneous system can be implemented by integrating the RF coil with a PET scanner or inserting the PET scanner inside a MRI. In these solutions, one of the challenges is to develop compact and magnetic field compatible PET detectors. Thanks to the SiPMs, such detectors now are realizable. Aim for the simultaneous PET/MRI system, we have developed a compact PET detector module by using of the SiPMs and MVT digitizers. In the module, output signals from the LYSO/SiPM detector blocks are sampled by the MVT digitizers and then sent out via the Ethernet communicator. The timing, energy and position information will be picked up by digitally analyzing the resulted samples in a PC. In the LYSO/SiPM detector blocks, we use cross-wire method to readout the signals from SiPMs and further using transmission-line method to multiplex the signals. In this way, we get a 36:4 readout channel reducing. In this work, we have setup a dual-panel coincidence detection and imaging system by using a pair of the detector modules. The timing and energy resolution of the system are measured to be $1.67 \mathrm{nS}$ FWMH and $16.8 \%$ @ $511 \mathrm{keV}$, respectively. With the system, we also obtained the imaging of a homemade mirco-Derenzo phantom which successfully resolved the $1.6 \mathrm{~mm}$ hollows. In future, we will investigate the feasibility of building a simultaneous PET/MRI system by using these modules.

\footnotetext{
Authors' details

${ }^{1}$ Huazhong University of Science \& Technology, Hubei, China. ${ }^{2}$ University of Chicago, Chicago, Illinois, USA.

Published: 18 May 2015
}

doi:10.1186/2197-7364-2-S1-A7

Cite this article as: Liu et al:: A compact PET detector module using SiPMs and MVT digitizers. EJNMMI Physics

2015 2(Suppl 1):A7.

\section{SpringerOpen $^{\odot}$}

(C) 2015 Liu et al; licensee Springer. This is an Open Access article distributed under the terms of the Creative Commons Attribution License (http://creativecommons.org/licenses/by/4.0), which permits unrestricted use, distribution, and reproduction in any medium, provided the original work is properly cited. 